EPJ Web of Conferences 85, 01005 (2015)

DOI: $10.1051 /$ epjconf/ 20158501005

(C) Owned by the authors, published by EDP Sciences, 2015

\title{
Status of DVMP, DVCS and GPDs
}

\author{
P. Kroll ${ }^{1, a}$ \\ ${ }^{1}$ Fachbereich Physik, Universität Wuppertal, 42097 Wuppertal, Germany and Institute für Theoretische Physik, Universität Re- \\ gensburg, 93040 Regensburg, Germany
}

\begin{abstract}
The analysis of exclusive meson leptoproduction (DVMP) within the handbag approach is reviewed and the parametrization of the generalized parton distributions (GPDs) is discussed in some detail with the main interest focused of the GPDs $H$ and $E$. Applications of the GPDs extracted from DVMP to other hard exclusive processes as for instance deeply virtual Compton scattering (DVCS) and an evaluation of Ji's sum rule are also presented.
\end{abstract}

\section{Introduction}

The handbag approach to hard exclusive leptoproduction of photons and mesons off protons has extensively been studied during the last fifteen years. It turned out that the handbag approach allows for a detailed analysis of cross sections, asymmetries and spin density matrix elements (SDME) for these processes. The handbag approach is based on factorization of the process amplitudes in a hard subprocess, e.g. $\gamma^{*} q \rightarrow \gamma(M) q$, and soft hadronic matrix elements parametrized in terms of GPDs. This factorization property has been shown to hold rigorously in the collinear limit for large photon virtuality, $Q$, and large energy, $W$, but fixed Bjorken- $x, x_{B}[1,2]$. However, power corrections to these asymptotic results are not under control. It is therefore unclear at which values of $Q^{2}$ and $W$ the asymptotic results apply. In fact, there are strong effects in meson leptoproduction which are not in accord with the asymptotic predictions. Thus, for instance, the contribution from longitudinally polarized virtual photons to likewise polarized vector (or pseudosalar) mesons transitions $\left(\gamma_{L}^{*} \rightarrow V_{L}(P)\right)$ dominate asymptotically; the ratio of the longitudinal and transverse cross sections $\left(R=\sigma_{L} / \sigma_{T}\right)$ grows proportionally to $Q^{2}$. Experimentally [3], $R$ for $\rho^{0}$ production only amounts to about 2 for $Q^{2} \lesssim 10 \mathrm{GeV}^{2}$, i.e. contributions from transversely polarized photons are not small. For $\omega$ production transverse photons even dominate [4], $R(\omega)$ is only about 0.3 for $2 \mathrm{GeV}^{2} \lesssim Q^{2} \lesssim 4 \mathrm{GeV}^{2}$. For $\pi^{0}$ production transverse photons probably dominate as well [5]. The amplitudes for $\gamma_{L}^{*} \rightarrow \rho_{L}^{0}$ transitions do also not plainly agree with the asymptotic picture which predicts the scaling law $\sigma_{L} \propto 1 / Q^{6}$ (modulo powers of $\ln Q^{2}$ from evolution and the running of $\alpha_{s}$ ) at fixed Bjorken$x$. As can be seen from Fig. 1 the data ${ }^{1}$ for the $\rho^{0}$ cross section [3] rather fall as $\lesssim 1 / Q^{4}$. Another example of corrections to the asymptotic results for the $\gamma_{L}^{*} \rightarrow V_{L}(P)$ am-

\footnotetext{
ae-mail: kroll@physik.uni-wuppertal.de

${ }^{1}$ Since $R$ is slightly increasing with $Q^{2} \sigma_{L}$ is even flatter than $1 / Q^{4}$.
}

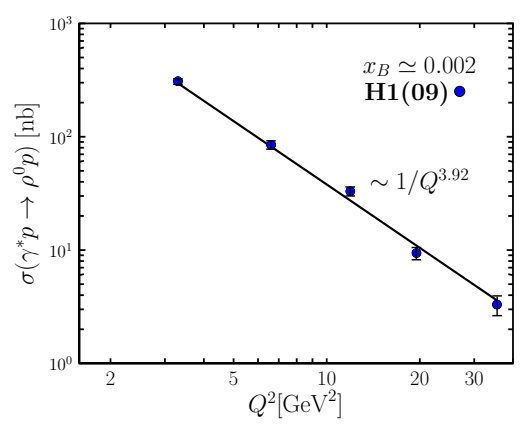

Figure 1. The cross section for $\rho^{0}$ electroproduction versus $Q^{2}$ at $x_{B} \simeq 0.002$. Data are taken from [3] and compared to a powerlaw fit.

plitudes is set by the strong contributions from the pion pole to $\pi^{+}$production that has been observed experimentally $[6,7]$. In this talk I am going to report on an extraction of the GPDs from DVMP [5, 8-10]. In this analysis the GPDs are constructed from double distributions (DDs) $[11,12]$ and the partonic subprocesses are computed within the modified perturbative approach in which quark transverse degrees of freedom as well as Sudakov suppression [13] are taken into account in order to model power corrections. As explained above these corrections are needed for instance in order to change the asymptotic $1 / Q^{6}$ fall of the longitudinal $\rho^{0}$ cross section in an effective $1 / Q^{4}$ behavior. The emission and re-absorption of the partons by the protons are treated collinearly to the proton momenta in [5, 8-10]. From the analyses of the longitudinal cross sections for $\rho^{0}$ and $\phi$ production the GPD $H$ has been extracted [8]. The transverse target spin asymmetries for $\rho^{0}$ production provide information on the GPD $E$. Generalizations of the handbag approach to $\gamma_{T}^{*} \rightarrow V_{T}$ and $\gamma_{T}^{*} \rightarrow V_{L}(P)$ transitions allow for a study of further GPDs $\left(\widetilde{H}, \widetilde{E}, H_{T}, \bar{E}_{T}\right)$. The extracted set of GPDs are sub- 
sequently be applied to calculate other hard exclusive processes free of adjustable parameters. An example of such an application is DVCS. Most of the observables of this process are under control of the GPD $H$. Nevertheless, the transverse target spin asymmetries in DVCS provide an additional constraint on $E$. The extracted GPDs $H$ and $E$ allow for an evaluation of the angular momenta the partons inside the proton carry. In the following sections these analyses and studies are described in some detail.

\section{The double distribution representation}

There is an integral representation of the GPDs in terms of DDs $[11,12]$ :

$$
\begin{aligned}
K^{i}(x, \xi, t) & =\int_{-1}^{1} d \rho \int_{-1+|\rho|}^{1-|\rho|} d \eta \delta(\rho+\xi \eta-x) r_{i}(\rho, \eta, t) \\
& +D_{i}(x, t) \Theta\left(\xi^{2}-x^{2}\right)
\end{aligned}
$$

where $K^{i}$ is some GPD. According to Diehl and Ivanov [14] there is an additional factor $x / \rho$ in (1) for $\widetilde{H}^{g}$ and $\widetilde{E}^{g}$. The last term in (1) is the so-called $D$-term [15] which appears for the GPDs $H$ and $E$. As a consequence of timereversal invariance the gluonic $D$-term is an even function of $x$ and the quark one an odd function. The advantage of the DD representation is that polynomiality of the GPDs is automatically satisfied.

A frequently used ansatz for the DD, $r_{i}$, associated with $K^{i}(x, \xi, t)$ is [16]

$$
r_{i}(\rho, \eta, t)=K^{i}(\rho, \xi=0, t) w_{i}(\rho, \eta) .
$$

The weight function, $w_{i}$, that generates the skewness dependence of the GPD, is assumed to be

$$
w_{i}(\rho, \eta)=\frac{\Gamma\left(2 n_{i}+2\right)}{2^{2 n_{i}+1} \Gamma^{2}\left(n_{i}+1\right)} \frac{\left[(1-|\rho|)^{2}-\eta^{2}\right]^{n_{i}}}{(1-|\rho|)^{2 n_{i}+1}}
$$

(in [8-10]: $n=1$ for valence quarks and 2 for sea quarks and gluons). The zero-skewness GPD for $\rho \geq 0$ is parametrized as its forward limit, $K^{i}(\rho, \xi=t=0)=k^{i}(\rho)$, multiplied by an exponential in Mandelstam $t$

$$
K^{i}(\rho, \xi=0, t)=k^{i}(\rho) \exp \left[t f_{i}(\rho)\right]
$$

and is to be suitably continued to negative $\rho$. For $H, \widetilde{H}$ and the transversity GPD $H_{T}$ the forward limits are the corresponding unpolarized, polarized and transversity parton distributions (PDFs), respectively ${ }^{2}$. The forward limits of the other GPDs $\left(E, \widetilde{E}, \bar{E}_{T}\right)$ which are not accessible in deep inelastic lepton-nuleon scattering (DIS), are parametrized in a fashion analogously to the PDFs

$$
k^{i}(\rho)=N_{i} \rho^{-\delta_{i}}(1-\rho)^{\beta_{i}}
$$

with free parameters $N_{i}, \delta_{i}$ and $\beta_{i}$ to be adjusted to data on exclusive reactions. In order to perform the DD integral (1) analytically the PDFs are expanded (for $H, \widetilde{H}, H_{T}$ ):

$$
k^{i}(\rho)=\rho^{-\delta_{i}}(1-\rho)^{2 n_{i}+1} \sum_{j=0}^{3} c_{i j} \rho^{j / 2} .
$$

\footnotetext{
${ }^{2}$ Note that by definition the forward limits of the gluonic GPDs have an extra factor of $x$, e.g. $H^{g}(x, \xi=t=0)=x g(x)$.
}

For quarks $\delta_{i}$ equals a Regge-like intercept $\alpha_{i}$ while, for gluons, $\delta_{g}=\alpha_{g}-1$ where $\alpha_{g}$ is a Pomeron-like intercept.

The profile function, $f_{i}(\rho)$, is parametrized in a Reggelike manner

$$
f_{i}(\rho)=-\alpha_{i}^{\prime} \ln \rho+B_{i}
$$

where $\alpha_{i}^{\prime}$ can be regarded as the slope of an appropriate Regge trajectory and $B$ parametrizes the $t$ dependence of its residue. This profile function is a simplified version of a more complicated one that has been proposed in $[17,18]$

$$
f_{i}(\rho)=\left(-\alpha_{i}^{\prime} \ln \rho+B_{i}\right)(1-\rho)^{3}+A_{i} \rho(1-\rho)^{2} .
$$

In order to elucidate the physics underlying the ansätze for the profile function let us consider the Fourier transform of the GPD $H^{q}(\rho, \xi=0, t)$ with respect to the momentum transfer $\boldsymbol{\Delta}_{\perp}$

$$
q\left(\rho, \mathbf{b}_{\perp}\right)=\int \frac{d^{2} \boldsymbol{\Delta}_{\perp}}{(2 \pi)^{2}} e^{-i \mathbf{b}_{\perp} \boldsymbol{\Delta}_{\perp}} H^{q}\left(\rho, \xi=0, t=-\Delta_{\perp}^{2}\right) .
$$

According to Burkardt $[19,20], q\left(\rho, \mathbf{b}_{\perp}\right)$ possess a density interpretation. The variable $\mathbf{b}_{\perp}$ is the transverse distance between the struck quark and the hadron's center of momentum defined by $\sum \rho_{j} \mathbf{b}_{\perp j}=0$. Evidently, quarks with a large momentum fraction $\rho_{j}$ must have a small transverse distance in that frame. In other words there is a correlation in $q\left(\rho, \mathbf{b}_{\perp}\right)$ between $\rho$ and $b_{\perp}$. In the limit $\rho \rightarrow 1 H^{q}$ becomes $t$ independent ${ }^{3}$. An estimate of the proton's transverse radius is provided by the average distance between the struck quark and the cluster of spectator partons:

$$
d_{q}(\rho)=\frac{\sqrt{\left\langle b_{\perp}^{2}\right\rangle_{\rho}^{q}}}{1-\rho}
$$

For the ansatz (4) the average distance reads

$$
d_{q}(\rho)=2 \frac{\sqrt{f_{q}(\rho)}}{1-\rho} .
$$

We see that the profile function (7) is singular for $\rho \rightarrow 1$ while (8) leads to $d_{q} \rightarrow 2 \sqrt{A_{q}}$ in this limit. The average distances for $u$ quarks obtained from both these profile functions are shown in Fig. 2. The Regge-like profile function is a reasonable approximation to (8) at small $\rho$.

At zero skewness $H^{q}$ exhibits a strong correlation between $\rho$ and $t$; the large $-t$ behavior of $H^{q}$ is under control of large $\rho$. The reason for this $\rho-t$ correlation in the parametrization of the GPDs described above, is easily understood. At small $\rho$ the PDF behaves $\sim \rho^{-\delta_{q}}$ with $\delta_{q} \simeq 0.5$ for valence quarks. For a simple $\rho-t$ factorized ansatz as has been used at the beginning of the handbag physics [21, 22]

$$
H^{q}(\rho, \xi=0, t) \sim q(\rho) F^{q}(t)
$$

the GPD possess that $\rho^{-\delta_{q}}$ singularity at all $t$. For the ansätze (7) and (8) the small $\rho$ behavior of the GPD is changed in

$$
H^{q}(\rho, \xi=0, t) \sim \rho^{-\left(\delta_{q}+\alpha_{q}^{\prime} t\right)} .
$$

\footnotetext{
${ }^{3}$ The profile function (7) don't possess this property except $B_{i}=0$.
} 


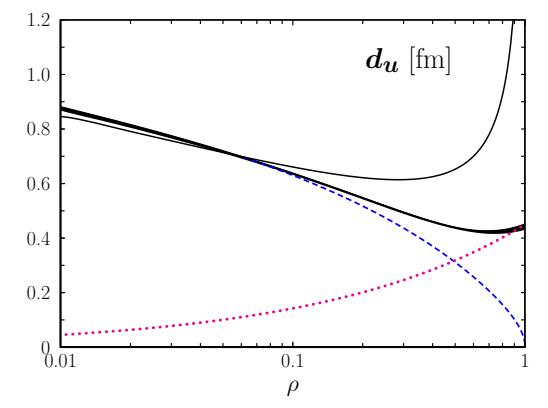

Figure 2. The distance $d_{u}$ evaluated from the profile functions (7) (dashed line) and (8) (solid line). The separate contributions from the low- $\rho$ (dashed) and the large- $\rho$ (dotted) term in the profile function (8) are also shown. The figure is taken from [18].

The $\rho^{-\delta_{q}}$ singularity occurring at $t=0$ becomes milder with increasing $-t$ and turns finally in a zero for $-t$ larger than $\delta_{q} / \alpha_{q}^{\prime}$. Given that for $-t \gtrsim \delta_{q} / \alpha_{q}^{\prime}$ the GPD, parametrized as in (4), only possesses nodes at the end points, it exhibits a pronounced maximum at a position that shifts towards higher $\rho$ with increasing $-t$. This property of the zero-skewness GPDs is transfered to the case of $\xi \neq 0$ through (1) although to a lesser degree. For all $\xi>0$ the GPD is peaked at a position $<\xi$ which increases with increasing $-t$. The peak becomes less pronounce with increasing $\xi$ (at fixed $t$ ) and with increasing $-t$ (at fixed $\xi$ ). Because of the $\rho-t$ correlation the profile function (7) can only be applied at small $-t$; it is unphysical at large $-t$.

In an alternative parametrization the GPDs are decomposed in terms of $t$-channel partial wave amplitudes. Each partial wave is parametrized in a Regge-like manner $[23,24]$.

\section{Extraction of the GPD $H$ from DVMP}

The asymptotically dominant $\gamma_{L}^{*} \rightarrow V_{L}$ amplitudes ${ }^{4}$ read $\left(V=\rho^{0}, \omega, \phi\right.$, the generalization to other vector mesons is straightforward)

$$
\begin{aligned}
& \mathcal{M}_{0+, 0+}^{V}=\frac{e_{0}}{2} \sqrt{1-\xi^{2}} \sum_{q=u, d, s} e_{q} C_{V}^{q}\left[\left\langle H_{\mathrm{eff}}^{g}\right\rangle_{V}+\left\langle H_{\mathrm{eff}}^{q}\right\rangle_{V}\right], \\
& \mathcal{M}_{0-, 0+}^{V}=-\frac{e_{0}}{2} \frac{\sqrt{t_{0}-t}}{2 m} \sum_{q=u, d, s} e_{q} C_{V}^{q}\left[\left\langle E^{g}\right\rangle_{V}+\left\langle E^{q}\right\rangle_{V}\right]
\end{aligned}
$$

where $e_{q}$ denote the quark charges in units of the positron charge, $e_{0}$, and $m$ the mass of the nucleon. Because of parity conservation it suffices to consider only the amplitudes with helicity $1 / 2$ of the initial state proton. The non-zero flavor weight factors read

$$
C_{\rho^{0}}^{u}=-C_{\rho^{0}}^{u}=C_{\omega}^{u}=C_{\omega}^{d}=1 / \sqrt{2}, \quad C_{\phi}^{s}=1 .
$$

The GPD $H_{\text {eff }}$ for quarks and gluons represents the combination

$$
H_{\mathrm{eff}}=H-\frac{\xi^{2}}{1-\xi^{2}} E
$$

\footnotetext{
${ }^{4}$ The light-cone helicities are labeled by their signs or by zero.
}
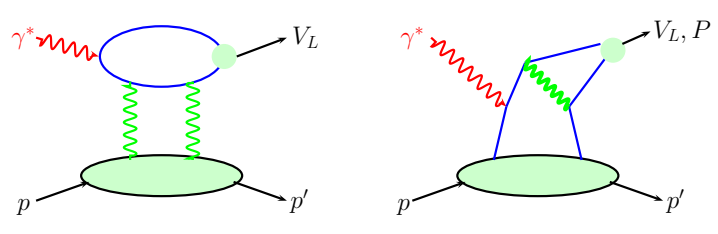

Figure 3. Typical leading-order Feynman graphs for exclusive meson production.

In [8] a meson-mass correction is taken into account in the relation between skewness and $x_{B}$

$$
\xi=\frac{x_{B}}{2-x_{B}}\left[1+m_{V}^{2} / Q^{2}\right] .
$$

The minimal value of $t$ allowed in the process is

$$
t_{0}=-4 m^{2} \frac{\xi^{2}}{1-\xi^{2}} .
$$

The convolutions $\langle K\rangle$ in (14) read

$$
\left\langle K^{i}\right\rangle_{V}=\sum_{\lambda} \int_{x_{i}}^{1} d x \mathcal{H}_{0 \lambda, 0 \lambda}^{V i}\left(x, \xi, Q^{2}, t=0\right) K^{i}(x, \xi, t)
$$

where $i=q, g$ and $x_{q}=-1, x_{g}=0$. The last item to be specified is the subprocess amplitude $\mathcal{H}$ for partonic helicity $\lambda$. In [8] it is calculated to leading-order of perturbation theory (see Fig. 3) taking into account quark transverse momenta, $\mathbf{k}_{\perp}$, in the subprocess and Sudakov suppressions. Since the latter involves a resummation of all orders of perturbation theory in next-to-leading-log approximation [13] which can only be efficiently performed in the impact-parameter space canonically conjugated to the $k_{\perp}$-space, one is forced to work in the $\mathbf{b}$-space. Hence,

$$
\begin{aligned}
\mathcal{H}_{0 \lambda, 0 \lambda}^{V i} & =\int d \tau d^{2} b \hat{\Psi}_{V}(\tau,-\mathbf{b}) \hat{F}_{0 \lambda, 0 \lambda}^{i}\left(x, \xi, \tau, Q^{2}, \mathbf{b}\right) \\
& \times \alpha_{s}\left(\mu_{R}\right) \exp \left[-S\left(\tau, \mathbf{b}, Q^{2}\right)\right] .
\end{aligned}
$$

$\hat{F}$ and $\hat{\Psi}$ are the Fourier transforms of the hard scattering kernel and the meson's light-cone wave, respectively. For the latter quantity a Gaussian in $\mathbf{b}$ is used with a parameter that describes the transverse size of the meson, and which is adjusted to experiment. $\mu_{R}$ is a suitable renormalization scale. The modified perturbative approach utilized in [8], is designed in such a way that asymptotically the leading-twist result [2] emerges. In passing I would like to remark that the treatment of the gluonic part of the amplitudes bears resemblance to the color-dipole model, see for instance [25-27].

In [8] the GPD $H$ is extracted from the data on the longitudinal cross section for $\rho^{0}$ and $\phi$ production in the kinematical region of small skewness and small $(\xi \lesssim 0.1$ and $-t \lesssim 0.5 \mathrm{GeV}^{2}$ ). In this kinematical situation the contributions from the GPD $E$ to the cross section can be ignored. The valence-quark GPDs $H$ at zero skewness are investigated in great detail in an analysis of the nucleon form factors [17] using the ansatz (4) with the profile function (8) and relying on the CTEQ6M(NLO) PDFs [28]. The resulting valence-quark GPDs in the small $-t$ approximation 


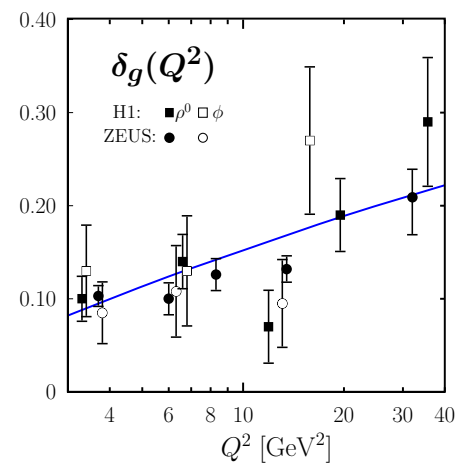

Figure 4. The intercept of the gluon trajectory $\delta_{g}=\alpha_{g}-1$ versus $Q^{2}$. Data are taken from Refs. [3, 30, 31]. The solid line represents the parametrization (24).

(7) are employed in [8]. It is checked that the GPDs from the updated version of the form factor analysis [18] which is based on the ABM11(NLO) PDFs [29] and includes all recent data of the nucleon form factors, entails only minor differences in the small $-t$ region. The CTEQ6M PDFs are also utilized for the gluon and sea quarks in [8]. The Regge-like parameters are assumed to be same for gluons and sea quarks, $\alpha_{g}=\alpha_{\text {sea }}$ and $\alpha_{g}^{\prime}=\alpha_{\text {sea }}^{\prime}$, since the sea-quark and gluon PDFs are strongly correlated by the evolution. At very small skewness as occur in the HERA experiments, the cross section behaves diffractively and is dominated by the imaginary part of the helicity non-flip amplitude, i.e.

$$
\sigma_{L} \propto\left|H^{g}(\xi, \xi, t \simeq 0)\right|^{2}
$$

where the mild shrinkage effect is ignored ${ }^{5}$. For the DD ansatz (1) one can show that

$$
H^{g}(\xi, \xi, t)=c\left(\delta_{g}, n_{g}, \alpha_{g}^{\prime} t\right) 2 \xi g(2 \xi) \mathrm{e}^{\left[B_{g}-\alpha_{g}^{\prime} \ln (2 \xi)\right] t} .
$$

Hence, at small skewness, $H^{g}(\xi, \xi, t \simeq 0) \sim \xi^{-\delta_{g}}$ with the consequence of a cross section obeying the power law

$$
\sigma_{L} \propto W^{4 \delta_{g}}
$$

at fixed values of $Q^{2}$. The parameter $\delta_{g}$ can therefore be read off from the HERA data $[3,30,31]$. The results are displayed in Fig. 4 and compared to the fit $\left(Q_{0}^{2}=4 \mathrm{GeV}^{2}\right)$

$$
\delta_{g}=0.10+0.06 \ln \left(Q^{2} / Q_{0}^{2}\right)-0.0027 \ln ^{2}\left(Q^{2} / Q_{0}^{2}\right) .
$$

For the slope of the gluon trajectory $\alpha_{g}^{\prime}$ a value of $0.15 \mathrm{GeV}^{-2}$ is taken. Thus, only the transverse size parameters in the wave functions for the $\rho^{0}$ and $\phi$ mesons as well as the parameter $B_{g}=B_{\text {sea }}$ in the profile function (7) have to be fitted to experiment. For more details of the GPD parametrizations see [8]. It is to be stressed that in [8] the evolution of the GPDs is approximated by that of the PDFs. Evolution is of importance only at large $Q^{2}$ which go along with large $W$ and small $\xi$ for the available

\footnotetext{
${ }^{5}$ With the above assumption on $\alpha_{\text {sea }}$ the sea quark contribution has the same energy dependence as the gluon one.
}

data. In this region the imaginary parts of gluon and sea quark contributions dominate, their real parts as well as the valence quark contribution are almost negligible. Because of (21) and (22) the approximate treatment of evolution is not unreasonable. However, an update of the analysis of the longitudinal cross sections for $\rho^{0}$ and $\phi$ production should not only include more recent sets of PDFS as for instance that of [29] but should also make use of the full GPD evolution as is incorporated in the code written by Vinnikov [32]. For a detailed study of the evolution of GPDs of the type discussed in Sect. 2, see [33].

In Figs. 5 and 6 a few of the results obtained in [8] are displayed. For $W>4 \mathrm{GeV}$ fair agreement between theory and experiment is to be seen for the longitudinal cross section of $\rho^{0}$ and $\phi$ production, integrated on $t$ from $t_{0}$ to $t_{0}-0.5 \mathrm{GeV}^{2}$. The error bands assigned to the theoretical results follow from the Hessian errors of the CTEQ6 PDFs. Exploiting other sets of PDFs (for instance $[34,35]$ ) one finds results which lie within the quoted error bands provided these PDFs are also fitted to the expansion (6) with the experimental value (24) of the power $\delta_{g}\left(Q^{2}\right)$. In contrast to $\phi$ production the handbag approach fails for $W$ below $\simeq 4 \mathrm{GeV}$ in the case of $\rho^{0}$ production. The strong increase of the data $[36,37]$ towards smaller $W$ is not reproduced. The kinematical region of $2 \mathrm{GeV} \leq W \leq 4 \mathrm{GeV}$ and $Q^{2} \simeq 4 \mathrm{GeV}^{2}$ is characterized by large skewness and large $-t_{0}$ (e.g. at $W=2 \mathrm{GeV}$ : $\xi=0.45$ and $t_{0}=-0.89 \mathrm{GeV}^{2}$ ). Thus, the use of the GPDs in that region requires an extrapolation from the region of $\xi \lesssim 0.1$ and $-t \lesssim 0.5 \mathrm{GeV}^{2}$ where the GPDs have been fixed, to the region of large $\xi$ and rather large $-t$. The behavior of the handbag results for the integrated cross sections at low $W$ reflects the decrease of the GPDs with $-t$, see (4). The dynamical origin of the experimentally observed behavior of the longitudinal cross section for $\rho^{0}$ production at low $W$ is unknown as yet. It has been conjectured in [39] that the $D$-term in (1) which has been neglected in [8], is responsible for it. However, it seems that this interpretation requires a large, nearly $t$-independent $D$ term. Both these properties seem to be in conflict with the findings in [40]. The cross sections for $\omega$ and $\rho^{+}$production behave similar to the $\rho^{0}$ cross section at low $W$. It is unclear whether all these processes can be described by the $D$-term in a consistent way.

In Fig. 6 the cross section for $\rho^{0}$ production is shown versus $Q^{2}$ at large $W$. In correspondence with Fig. 1 the unseparated cross section is displayed. Good agreement with experiment is seen for $Q^{2}$ ranging from about 4 to $100 \mathrm{GeV}^{2}$. The leading-twist result shown for comparison, deviates substantially from experiment at lower values of $Q^{2}$ but is close at $Q^{2} \simeq 100 \mathrm{GeV}^{2}$. This feature has already been discussed in the context of Fig. 1 . In the modified perturbative approach utilized in the computation of the subprocess amplitude [8], the Sudakov factor and the meson wave function generate series of power corrections of the type $\left(\Lambda_{\mathrm{QCD}} / Q\right)^{2 n}$ and $\left(k_{\perp} / Q\right)^{2 n}$, respectively. These power corrections reduce the leading-twist behavior of $\sigma$ from $\sim 1 / Q^{6}$ to an effective $1 / Q^{4}$ one which is in agreement with experiment. An alternative concept is 

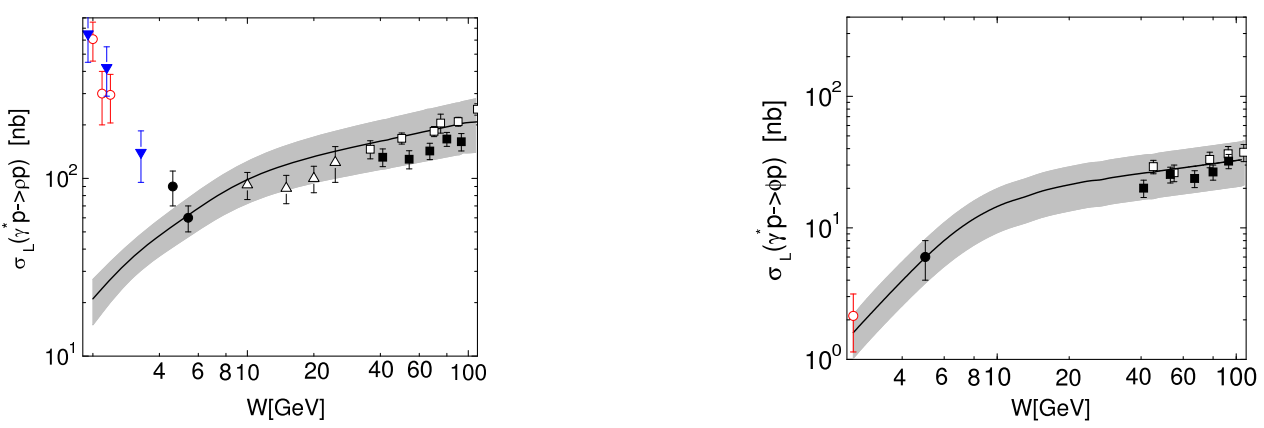

Figure 5. The longitudinal cross sections for $\rho^{0}$ and $\phi$ production at $Q^{2}=4 \mathrm{GeV}^{2}$ and $3.8 \mathrm{GeV}^{2}$, respectively. Data are taken from $[3,36-38]$, further references can be found in [8]. The solid lines with the error bands represent the results from the handbag approach. The H1 data [3] (solid squares) are not included in the fits performed in [8].

advocated for in [23]. Their GPD $H$, fitted to the HERA data on DVMP and DVCS in collinear approximation, exhibit strong evolution effects. I.e. the reduction from the $1 / Q^{6}$ fall to an effective $1 / Q^{4}$ one is realized by powers of $\ln Q^{2}$. It remains to be seen whether this concept can be extended to smaller $W$. It should be mentioned that in collinear approximation a fit to only the DVCS data and a fit to both DVCS and DVMP data lead to different GPDs [23].

\section{Generalizations and applications}

In [9] the handbag approach has been generalized to the amplitudes for $\gamma_{T}^{*} \rightarrow V_{T}$ transitions. In collinear approximation the subprocess amplitudes for such transitions are infrared singular. The quark transverse momenta which are taken into account in the modified perturbative approach, regularize these infrared singularities although in a model-dependent way. The transverse amplitudes are suppressed by $\left\langle k_{\perp}^{2}\right\rangle^{1 / 2} / Q$ with respect to the longitudinal ones. With the $\gamma_{T}^{*} \rightarrow V_{T}$ amplitudes at disposal the transverse cross sections as well as some of the SDMEs for $\rho^{0}$ and $\phi$ leptoproduction can be computed. Reasonable agreement of the $\gamma_{T}^{*} \rightarrow V_{T}$ amplitudes with experiment is found with the exception of the relative phase between the longitudinal and transverse amplitudes which appears to be somewhat small. An example of these results is shown in Fig. 6.

The handbag approach has also been generalized to the $\gamma_{T}^{*} \rightarrow V_{L}, P$ transition amplitudes $[5,10]$. The asymmetries measured with a transversely polarized target by the HERMES collaboration [41] for $\pi^{+}$electroproduction signal the importance of the helicity non-flip amplitude $\mathcal{M}_{0-,++}$ in that process. This amplitude is modeled by a convolution of the transversity GPD $H_{T}$ and the quark helicity-flip subprocess amplitude which necessitates the use of a twist-3 meson wave function [42, 43]:

$$
\mathcal{M}_{0-,++}^{\pi^{+}}=e_{0} \sqrt{1-\xi^{2}} \int_{-1}^{1} d x \mathcal{H}_{0-.++}^{\pi^{+}}\left(H_{T}^{u}-H_{T}^{d}\right) .
$$

This amplitude is parametrically suppressed by $\mu_{\pi} / Q$ with respect to the asymptotically leading $\gamma_{L}^{*} \rightarrow \pi^{+}$amplitudes which look like (14) for the appropriate flavor combination

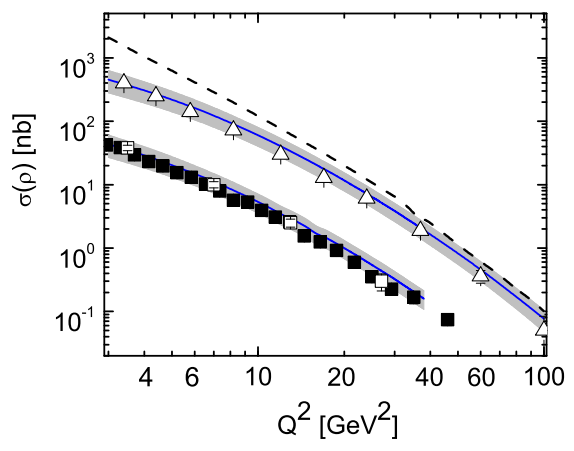

Figure 6. The integrated cross section for $\rho^{0}$ production versus $Q^{2}$ at $W=75$ (divided by 10 for the ease of legibility) and $90 \mathrm{GeV}$. For comparison the leading-twist result at $90 \mathrm{GeV}$ is also shown as a dashed line. The figure is taken from [9]. For further notations see Fig. 5.

with the replacement of $H_{\text {eff }}$ by $\widetilde{H}_{\text {eff }}$ and of $E$ by $\xi \widetilde{E}$. The parameter $\mu_{\pi}$ is large, $\simeq 2 \mathrm{GeV}$ at a scale of $2 \mathrm{GeV}$, since it is given by the pion mass, $m_{\pi}$, enhanced by the chiral condensate

$$
\mu_{\pi}=\frac{m_{\pi}^{2}}{m_{u}+m_{d}}
$$

by means of the divergency of the axial-vector current $\left(m_{u}\right.$ and $m_{d}$ denote current-quark masses).

A special feature of $\pi^{+}$production is the pion-pole which contributes to the GPD $\widetilde{E}[44,45]$

$$
\widetilde{E}^{\text {pole }}=\Theta(|x| \leq \xi) \frac{F_{P}^{\text {pole }}}{2 \xi} \Phi_{\pi}\left(\frac{x+\xi}{2 \xi}\right)
$$

where $\Phi_{\pi}$ is the pion distribution amplitude and $F_{P}^{\text {pole }}$ the contribution of the pion-pole to the pseudoscalar nucleon form factor. As already mentioned in the introduction the pion-pole contribution to $\pi^{+}$production fails by order of magnitude if estimated through $\widetilde{E}$ since it is proportional to the square of the pion electromagnetic form factor in onegluon exchange approximation. In [10] the pion pole is therefore treated as a one-particle exchange leading to the same result for its contribution to the cross section except that full experimental value of the pion form factor appears which is about a factor of 2 to 3 larger than the perturbative 


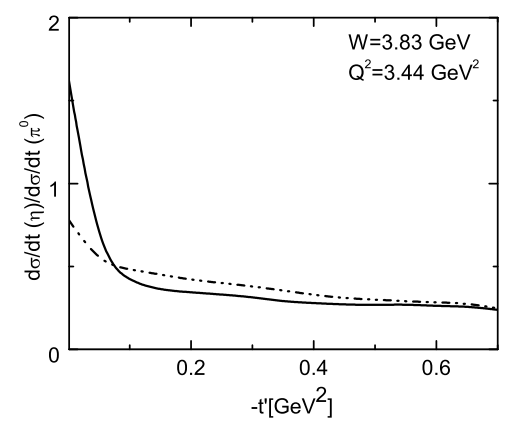

Figure 7. The ratio of the $\eta$ and $\pi^{0}$ cross sections versus $t^{\prime}=t-t_{0}$ for two different parametrizations of $H_{T}$ (see [5]).

result. The analysis of the HERMES data on $\pi^{+}$production $[6,41]$ leads to a determination of the GPDs $\widetilde{H}$ and $H_{T}$ for valence quarks of, however, a lesser quality than $H$. The present data do not allow for a reliable determination of a non-pole contribution to $\widetilde{E}$.

In $\pi^{+}$production the transversity GPD $\bar{E}=2 \widetilde{H}_{T}+E_{T}$ plays a minor role while it seems to be very important in $\pi^{0}$ production [5]. This GPD feds the amplitudes

$$
\mathcal{M}_{0+. \pm+}^{\pi^{+}}=-\frac{e_{0}}{2} \frac{\sqrt{t_{0}-t}}{2 m} \int_{-1}^{+1} d x \mathcal{H}_{0-,++}^{\pi^{+}}\left(\bar{E}_{T}^{u}-\bar{E}_{T}^{d}\right)
$$

with the same subprocess amplitude as in (25). The generalization of (25) and (28) to other pseudoscalar mesons and even to vector mesons [46] (where $\mu_{V}=m_{V}$ ) is straightforward. Lack of suitable data, e.g. the $\pi^{0}$ cross section at small skewness, prevents a determination of $\bar{E}_{T}$ from experiment as yet. In [5] it is estimated by fixing its parameters with the help of lattice QCD results on moments of $\bar{E}_{T}$ [47]. From this estimate interesting predictions for leptoproduction of pseudoscalar mesons are obtained. Thus, for instance, this GPD dominates $\pi^{0}$ production (leading for instance to $\sigma_{L} / \sigma_{T} \simeq 0.1$ for this process). Another example is the $\eta / \pi^{0}$ cross-section ratio which amounts to about $1 / 3$ for $t-t_{0}$ not too close to zero. This result is in sharp contrast to a leading-twist prediction of $\gtrsim 1$ [48]. Such a large $\eta / \pi^{0}$ ratio may hold at the best at $t-t_{0} \simeq 0$ where the GPD $\bar{E}_{T}$ does not contribute (see (28)) and the helicity non-flip amplitudes, under control of $\widetilde{H}$ and $H_{T}$, take the lead. Suppose there is a dominant GPD. The $\eta / \pi^{0}$ ratio is then given by

$$
\frac{d \sigma(\eta)}{d \sigma\left(\pi^{0}\right)} \sim\left(\frac{\left\langle e_{u} K^{u}+e_{d} K^{d}\right\rangle_{\eta}}{\left\langle e_{u} K^{u}-e_{d} K^{d}\right\rangle_{\pi^{0}}}\right)^{2} .
$$

Evidently, the relative sign and magnitude of the dominant GPDs for $u$ and $d$ quarks determine the value of the ratio. According to the lattice result [47] $\bar{E}_{T}^{u}$ and $\bar{E}_{T}^{d}$ have the same sign while $\widetilde{H}$ and $H_{T}$ have the opposite sign for $u$ and $d$ quarks as follows from the polarized and transversity PDFs $[49,50]$. Ignoring differences between the $\eta$ and the $\pi^{0}$ wave functions and, for a quick estimate, taking the $u / d$ ratio of the first moments of the zero-skewness GPD at $t=0$ as representative of the ratio of the convolutions, one finds the values 0.26 for a dominant $\bar{E}_{T}$ [47], 1.64 for
Table 1. Status of small-skewness GPDs as extracted from meson leptoproduction data. At present no information is available on GPDs not appearing in the list. Except of $H$ for gluons and sea quarks all GPDs are only probed for scales of about $4 \mathrm{GeV}^{2}$. For comparison five stars are assigned to PDFs.

\begin{tabular}{|c||c|c|c|}
\hline GPD & probed by & constraints & status \\
\hline$H($ val $)$ & $\rho^{0}, \phi$ cross sect. & PDFs, Dirac $\mathrm{ff}$ & $* * *$ \\
$H(\mathrm{~g}, \mathrm{sea})$ & $\rho^{0}, \phi$ cross sect. & PDFs & $* * *$ \\
$E($ val $)$ & $A_{U T}\left(\rho^{0}, \phi\right)$ & Pauli ff & $* *$ \\
$E(\mathrm{~g}, \mathrm{sea})$ & - & $(42)$ & - \\
$\widetilde{H}(\mathrm{val})$ & $\pi^{+}$data & pol. PDFs, axial $\mathrm{ff}$ & $* *$ \\
$\widetilde{H}(\mathrm{~g}, \mathrm{sea})$ & $A_{L L}\left(\rho^{0}\right)$ & polarized PDFs & $*$ \\
$\widetilde{E}(\mathrm{val})$ & $\pi^{+}$data & pseudoscalar $\mathrm{ff}$ & $*$ \\
$H_{T}(\mathrm{val})$ & $\pi^{+}$data & transversity PDFs & $*$ \\
$\bar{E}_{T}(\mathrm{val})$ & $\pi^{+}$data & - & $*$ \\
\hline
\end{tabular}

a dominant $H_{T}$ [50] and 2.40 for the case of $\widetilde{H}$ [49] for the $\eta / \pi^{0}$ cross-section ratio. This is what one observes from Fig. 7. Large skewness data from CLAS $[51,52]$ are in agreement with these findings: the $\eta / \pi^{0}$ ratio is $\simeq 0.3$ for $-t \geq 0.1 \mathrm{GeV}^{2}$.

A summary of the information about the GPDs extracted from DVMP is given in Tab. 1. With these GPDs at disposal one is in the position to calculate observables for other hard exclusive processes exploiting the universality property of the GPDs. Thus, in [53] neutrino induced exclusive pion production has been computed. Due to the parity-violating $V-A$ structure of the electro-weak interactions the GPDs $H$ and $E$ also contribute to $v_{l} p \rightarrow l p \pi$. There are no data available at present but this process may be relevant for the MINERVA experiment FERMI LAB. Another example is time-like DVCS for which predictions, again evaluated from this set of GPDs, have been given in $[54,55]$ recently. It would be interesting to compare them with data. In [56] exclusive leptoproduction of the $\omega$ meson has been computed and compared to the SDMEs measured by HERMES [4]. Fair agreement is found. An important element in this calculation is the pion-pole. As in [10] it has been treated as an one-particle exchange; its calculation through (27) underestimates the effect as is the case for $\pi^{+}$production. Instead of the electromagnetic form factor of the pion in the latter process the $\pi \omega$ transition form factor occurs now on which information at rather large $Q^{2}$ has been extracted in [56]. The pion pole dominantly contributes to the $\gamma_{T}^{*} \rightarrow \omega_{T}^{*}$ and $\gamma_{L}^{*} \rightarrow \omega_{T}^{*}$ transition amplitudes which are suppressed by $1 / Q$ and $1 / Q^{2}$ with respect to the asymptotically leading $\gamma_{L}^{*} \rightarrow \omega_{L}^{*}$ amplitudes, respectively. As a consequence $\omega$ production looks very different from the asymptotic picture at $W \simeq 5 \mathrm{GeV}: \sigma_{T}>\sigma_{L}$ and the unnatural parity cross section is larger than the natural-parity one. As an example for the strength of the unnatural-parity contribution the ratio $U_{1}=d \sigma^{U} / d \sigma$ is shown in Fig. 8. Since the $\pi \rho^{0}$ transition form factor is about third of the $\pi \omega$ one [57] the pion-pole contribution to leptoproduction of the $\rho^{0}$ is rather small but larger than what is obtained from (27). It 


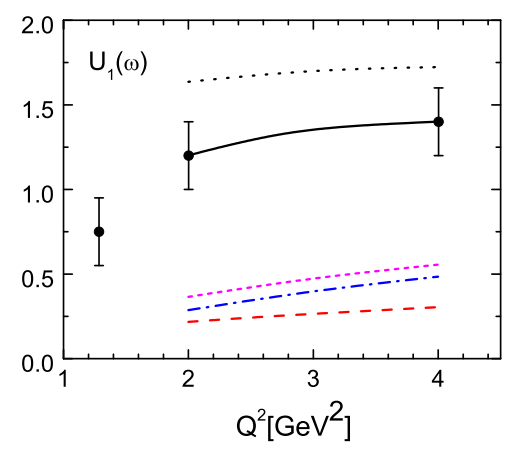

Figure 8. The unnatural-parity cross section for $\omega$ leptoproduction at $W=4.8 \mathrm{GeV}$ and $t-t_{0}=-0.08 \mathrm{GeV}^{2}$. The solid (longdashed) line represents the handbag result for $U_{1}$ with (without) the pion pole. Data are taken from [4]. For other notation see [56].
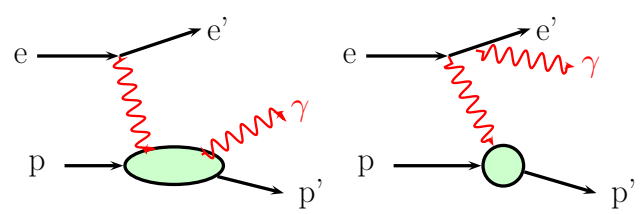

Figure 9. Typical leading-order Feynman graphs for electroproduction of photons.

has hardly to be seen in most of the observables for $\rho^{0}$ production. Exceptions are for instance the unnatural-parity cross section and the relative phase between the longitudinal and transverse amplitudes which is enlarged from $3.1^{\circ}$ to $13.4^{\circ}$. The inclusion of the pion pole brings both these quantities closer to the experimental results [58].

\section{DVCS}

Another important application of the set of GPDs extracted in [8-10] is the evaluation of DVCS. This task has been performed by two groups: by the authors of [59] and by Kumericky et al. published in [60]. Typical Feynman graphs for a leading-order calculation of leptoproduction of photons are shown in Fig. 9. As is well-known there are two contributions to this process - the Bethe-Heitler (BH) contribution for which the photon is emitted from the lepton and the proper DVCS contribution where the photon is emitted from the proton. Evidently, for a collinear emission and re-absorption of quarks from the protons a quark transverse momentum is impossible in the subprocess $\gamma^{*} q \rightarrow \gamma q$. Therefore, the use of the collinear approximation for DVCS is consistent with the treatment of DVMP as described in Sect. 3.

The square of the amplitude for leptoproduction of real photons consists of three terms

$$
|\mathcal{T}(l p \rightarrow l p \gamma)|^{2}=|\mathcal{T}(\mathrm{BH})|^{2}+\mathcal{T}_{\mathrm{I}}+|\mathcal{T}(\mathrm{DVCS})|^{2}
$$

where $\mathcal{T}_{I}=2 \mathcal{T}(\mathrm{BH}) \operatorname{Re} \mathcal{T}(\mathrm{DVCS})$. The Bethe-Heitler contribution can be worked out without any approximation [61] and is related to the electromagnetic form factors of the nucleon. The c.m.s. $\gamma^{*} p \rightarrow \gamma p$ amplitudes to leadingorder of perturbation theory read

$$
\begin{aligned}
& \mathcal{M}_{ \pm+, \pm+}\left(\gamma^{*} p \rightarrow \gamma p\right)=\frac{e_{0}^{2}}{2} \sqrt{1-\xi^{2}}\left[\left\langle H_{\mathrm{eff}}\right\rangle_{\gamma} \pm\left\langle\widetilde{H}_{\mathrm{eff}}\right\rangle_{\gamma}\right], \\
& \mathcal{M}_{ \pm-, \pm+}\left(\gamma^{*} p \rightarrow \gamma p\right)=-\frac{e_{0}^{2}}{2} \frac{\sqrt{t_{0}-t}}{2 m}\left[\langle E\rangle_{\gamma} \pm\langle\widetilde{E}\rangle_{\gamma}\right],
\end{aligned}
$$

(compare with (14)) and involve the convolutions

$$
\begin{aligned}
\langle K\rangle_{\gamma} & =\int_{-1}^{1} d x\left[e_{u}^{2} K^{u}+e_{d}^{2} K^{d}+e_{s}^{2} K^{s}\right] \\
& \times\left[\frac{1}{\xi-x-i \varepsilon}-\epsilon_{k} \frac{1}{\xi+x-i \varepsilon}\right]
\end{aligned}
$$

where $\epsilon_{k}=+1$ for $K=H, E$ and -1 for $\widetilde{H}, \widetilde{E}$.

The three terms in (30) have the following harmonic structure in $\phi$, the azimuthal angle of the outgoing photon with regard to the leptonic plane ( $\mathrm{i}=\mathrm{BH}, \mathrm{DVCS})$ :

$$
\left|\mathcal{T}_{i}\right|^{2} \propto L_{i} \sum_{n=0}^{3}\left[c_{n}^{i} \cos (n \phi)+s_{n}^{i} \sin (n \phi)\right]
$$

where $L_{\mathrm{BH}}=[-t P(\cos \phi)]^{-1}$ and $L_{\mathrm{DVCS}}=1$. An analogous Fourier series holds for the interference term. Although there are only harmonics up to the maximal order 3 in the sums, the additional $\cos \phi$ dependence from the lepton propagators, included in $P(\cos \phi)$, generates in principle an infinite series of harmonics for the $\mathrm{BH}$ and interference terms. A more detailed harmonic structure taking into account beam and target polarizations can be found in [62].

A comparison of this theoretical approach with experiment performed in [59], reveals reasonable agreement with HERMES, H1 and ZEUS data and a less satisfactory description of the large-skewness, small $W$ JLab6 data. As discussed in Sect. 3 the application of the GPDs extracted from DVMP at JLab6 kinematics is problematic: It requires an extrapolation in $\xi$ and $t$, one has to be aware of possible soft-physics contributions (see e.g. Fig. 5) as well as of large kinematical corrections. For example, in the relation (17) there are additional terms proportional to $x_{B}$, e.g. $x_{B} t / Q^{2}$ which are negligible in the small $\xi,-t$ region but not at JLab6 kinematics [63].

It turns out that most of the $l p \rightarrow l p \gamma$ observables are under control of the best determined GPD $H$ (see Sect. 3 and Tab. 1), only a few observables are sensitive to $E$ (e.g. particular modulations of $A_{U T}$ ) and $\widetilde{H}$ (e.g. the $\sin \phi$ modulation of $\left.A_{U L}\right)$. The GPD $\widetilde{E}$ does not plays a role in DVCS in practice.

The DVCS cross section at HERA kinematics is shown in Fig. 10. Given that this is a parameter-free calculation the agreement with experiment $[64,65]$ is impressive. Similar results have been obtained in [23].

The $l p \rightarrow l p \gamma$ cross section on an unpolarized target for a given beam charge, $e_{l}$, and beam helicity, $h_{l} / 2$, can be decomposed as

$$
\begin{aligned}
d \sigma^{h_{l}, e_{l}}(\phi) & =d \sigma_{\mathrm{UU}}(\phi)\left[1+h_{l} A_{\mathrm{LU}, \mathrm{DVCS}}(\phi)\right. \\
& \left.+e_{l} h_{l} A_{\mathrm{LU}, \mathrm{I}}(\phi)+e_{l} A_{C}[\phi)\right]
\end{aligned}
$$




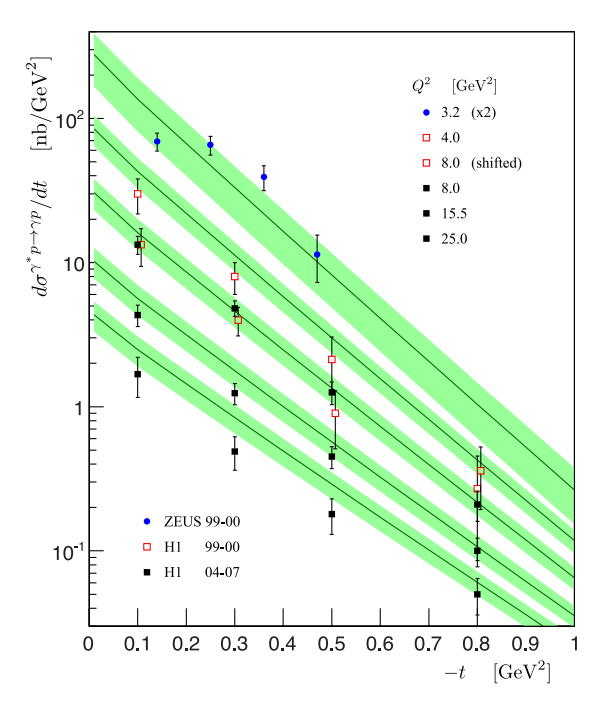

Figure 10. The DVCS cross section for various values of $Q^{2}$ and $W$ ranging between 71 and $104 \mathrm{GeV}$. Data are taken from $[64,65]$. The results obtained in [59] are shown as solid lines with error bands.

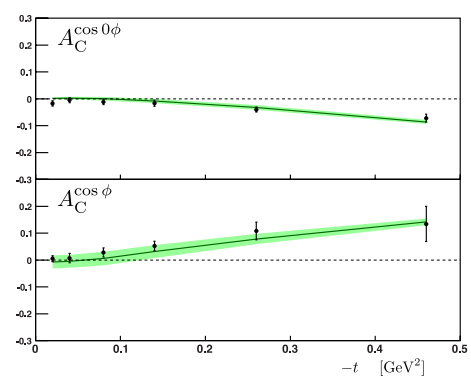

Figure 11. The beam charge asymmetry versus $-t$ at $Q^{2} \simeq$ $2.51 \mathrm{GeV}^{2}, x_{B} \simeq 0.097$. Data are taken from [66]. For further notations see Fig. 10 .

where only the $\phi$-dependence of the observables is made explicit. If a longitudinally polarized beam of both lepton charges is available the asymmetries in (34) can be isolated. Two modulations of the beam charge asymmetry, $A_{C}$, are shown in Fig. 11. They are under control of the BH-DVCS interference and depend mostly on $\operatorname{Re}\langle H\rangle_{\gamma}$. The agreement of the handbag results with the HERMES data [66] demonstrates that the convolution of $H$ has the right magnitude.

The HERMES collaboration has measured the $\sin \phi$ modulation of the beam spin asymmetry using a recoil detector [67] which allows for a detection of all three final-state particles. The resonant background is therefore severely reduced and in so far the recoil data are closer to the exclusive process $l p \rightarrow l p \gamma$ to which the theory applies. Since the recoil data are available only for a positron beam the observables defined in (34) cannot be isolated. In fact, the combination

$$
A_{\mathrm{LU}}^{+\sin \phi} \simeq \frac{A_{\mathrm{LU}, \mathrm{I}}^{\sin \phi}}{1+A_{C}^{\cos (0 \phi)}}
$$

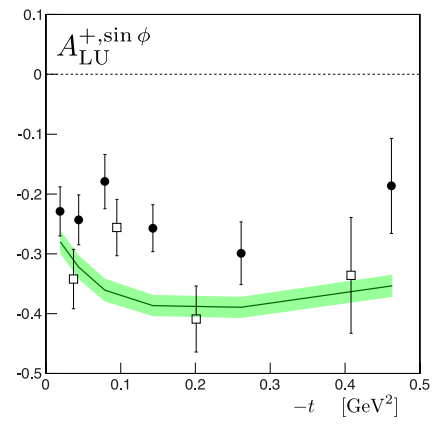

Figure 12. The $\sin \phi$ modulation of $A_{L U}^{+}$versus $-t$ at $Q^{2} \simeq$ $2.5 \mathrm{GeV}^{2}, x_{B} \simeq 0.09$. Data are taken from [66] (solid circles) and [67] (open squares). For further notations see Fig. 10.

is measured by the HERMES collaboration. In (35) a contribution from $A_{\mathrm{LU}, \mathrm{DVCS}}$ is neglected which is exactly zero at twist-2 accuracy in agreement with experiment [66]. Since $A_{C}^{\sin (0 \phi)}$ is so small $A_{\mathrm{LU}}^{+, \sin \phi}$ and $A_{\mathrm{LU}, \mathrm{I}}^{\sin \phi}$ don't differ much. As can be seen from Fig. 12 the handbag results for $A_{\mathrm{LU}}^{+\sin \phi}$ agree quite well with the recoil data while the agreement with the non-recoil data [66] is surprisingly bad. Recoil data for other observables would be welcome.

Results on the transverse target spin asymmetries will be discussed in the next section.

\section{The GPD $E$}

Next let me discuss the GPD $E$ in some detail. The analysis of the nucleon form factors performed in [17] and updated in [18], provides the zero-skewness GPDs for valence quarks which can be used as input to the DD representation (1). The basis of this analysis are the sum rules for the flavor form factors

$$
\begin{aligned}
& F_{1}^{q}(t)=\int_{0}^{1} d \rho H_{v}^{q}(\rho, \xi=0, t), \\
& F_{2}^{q}(t)=\int_{0}^{1} d \rho E_{v}^{q}(\rho, \xi=0, t)
\end{aligned}
$$

where the valence quark GPDs are defined by

$$
K_{v}^{q}(\rho, \xi=0, t)=K^{q}(\rho, \xi=0, t)+K^{q}(-\rho, \xi=0, t) .
$$

The Dirac $(i=1)$ and Pauli $(i=2)$ form factors for the proton and the neutron are decomposed in the flavor form factors as

$$
F_{i}^{p}=e_{u} F_{i}^{u}+e_{d} F_{i}^{d}+e_{s} F_{i}^{s}, \quad F_{i}^{n}=e_{u} F_{i}^{d}+e_{d} F_{i}^{u}+e_{s} F_{i}^{s} .
$$

Estimates say that the strangeness form factors can be neglected, their contributions are at most of the size of the errors on $F_{i}^{u}$ and $F_{i}^{d}$. The valence-quark GPDs are parametrized as in (4) and (8). The forward limits of $E$ are parametrized as in (5); in [18] an additional factor $\left(1+\gamma_{q} \sqrt{\rho}\right)$ is used with $\gamma_{u}=4$ and $\gamma_{d}=0$. This factor improves $\chi^{2}$ slightly. The GPD $E$ is constrained by

$$
\int_{0}^{1} d \rho e_{v}^{q}(\rho)=\kappa_{q}
$$



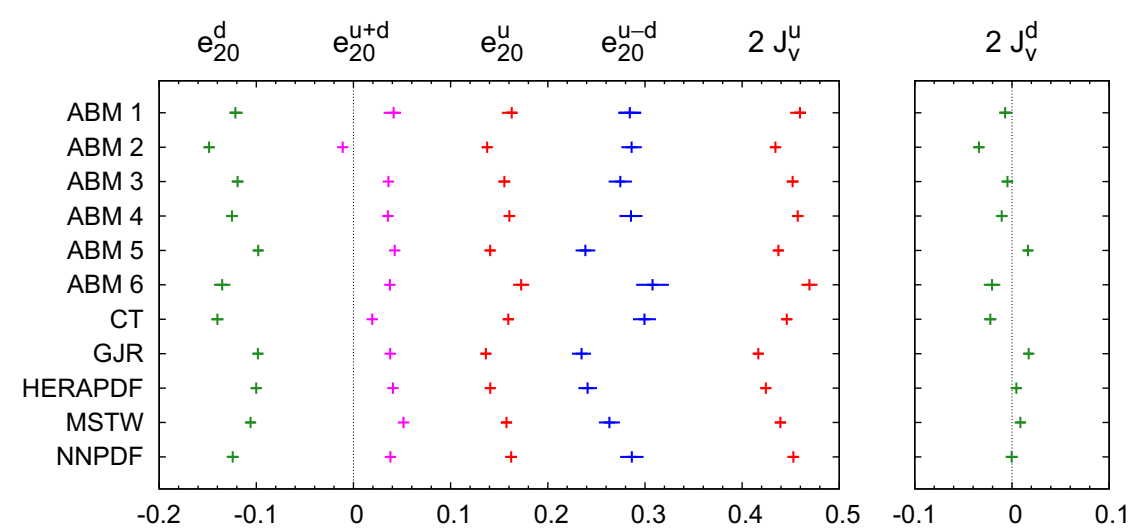

Figure 13. Results for the second moments of $E$ and the angular momenta of the valence quarks at a scale of $2 \mathrm{GeV}$. Shown is the default fit ABM1 and variations with regard to the strangeness form factors, other data interpolations and different sets of PDFs [18].
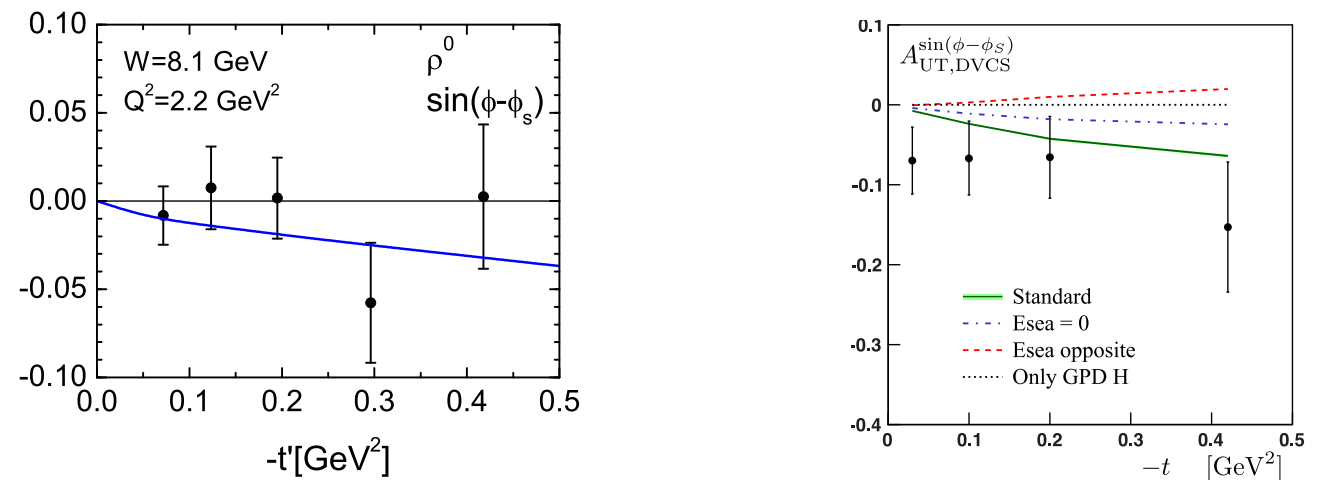

Figure 14. Left (right): The $\sin \left(\phi-\phi_{s}\right)$ modulation of $A_{\mathrm{UT}}$ for $\rho^{0}$ production (DVCS). Data are taken from [77] ([78]), the theoretical results from [46] ([59]).

where $\kappa_{q}$ is the contribution of quarks of flavor $q$ to the anomalous magnetic moments of the nucleon $\left(\kappa_{u}=1.67\right.$, $\left.\kappa_{d}=-2.03\right)$. In Sect. 3 it has already been remarked that the 2004 analysis [17] is based on the CTEQ6M PDFs [28] while the recent update [18] uses the ABM11 PDFs [29] for the default fit. Since in 2004 data on the neutron form factors were only available for $-t \lesssim 2 \mathrm{GeV}^{2}$ the parameters of the zero-skewness GPD $E$ were not well fixed; a particularly wide range of values were allowed for the powers $\beta_{e}^{u}$ and $\beta_{e}^{d}$. In the reanalysis [18] use is made of the new data on the neutron form factors and the ratio of electric and magnetic proton form factors which extend to much larger values of $-t$ than before [68-71]. Because of the $\rho-t$ correlation discussed above, the powers $\beta_{e}^{q}$ are better determined now $\left(\beta_{u}=4.65, \beta_{d}=5.25, \alpha_{u}(0)=\alpha_{d}(0)=0.603\right)$. At small $-t$ the new results for the valence-quark GPDs are similar to the 2004 version. As an example of the results derived in [18] the second moments of $E$ for valence quarks at $\xi=t=0$ are displayed in Fig. 13.

For the following discussion it is convenient to change a little bit the notation. In analogy to the PDFs we define

$$
\begin{aligned}
e^{q}(\rho) & =E_{q}(\rho, \xi=t=0), e^{\bar{q}}(\rho)=E_{q}(-\rho, \xi=t=0) \\
\rho e^{g}(\rho) & =E_{g}(\rho, \xi=t=0)
\end{aligned}
$$

The Mellin moments are defined as

$$
e_{20}^{q}=\int_{0}^{1} d \rho \rho^{n-1} e^{q}(\rho), \quad e_{20}^{g}=\int_{0}^{1} d \rho \rho^{n-1} e^{g}(\rho),
$$

and analogously for the PDFs.

Not much is known about $E^{g}$ and $E^{\text {sea }}$. There is only a sum rule for the second moments of $E$ [72] at $t=\xi=0$

$$
e_{20}^{g}=-\sum e_{20}^{q_{v}}-2 \sum e_{20}^{\bar{q}}
$$

It turns out that the valence contribution to the sum rule is very small. In fact, $e_{20}^{u_{v}}+e_{20}^{d_{v}}=0.041_{-0.053}^{+0.011}$ for the default fit, see Tab. 18 in [18]. Hence, the second moments of the gluon and sea-quark GPD $E$ cancel to a large extent. Since the parametrization (5) for the forward limit of $E$ does not have nodes except at the end-points this property approximately holds for other moments as well and even for the convolution (19).

A further information about $E$ for strange quarks comes from a positivity bound for its Fourier transform [73]:

$$
\frac{b_{\perp}^{2}}{m^{2}}\left(\frac{\partial e_{s}\left(\rho, b_{\perp}\right)}{\partial b_{\perp}^{2}}\right) \leq s^{2}\left(\rho, b_{\perp}\right)-\Delta s^{2}\left(\rho, b_{\perp}\right)
$$

where $s, \Delta s$ and $e_{s}$ are the Fourier transforms of the zeroskewness GPDs $H^{s}, \widetilde{H}^{s}$ and $E^{s}$, respectively. As shown in 
$[74,75]$ this bound forbids a large strange quark contribution and, assuming a flavor-symmetric sea, a large gluon contribution too. The bound on $e^{s}\left(\rho, b_{\perp}\right)$ is saturated for $N_{e}^{s}= \pm 0.155\left(\beta_{e}^{s}=7\right.$ and the same $\delta_{s}$ as for $H^{s}$ in (5)) [75]. The normalization of $e^{g}$ can subsequently be fixed from the sum rule (42) $\left(\beta_{e}^{g}=6\right.$ and the same $\delta_{g}$ as for $\left.H^{g}\right)$. These results are inserted in (1) in order to obtain estimates of $E^{\text {sea }}$ and $E^{g}$. Diehl in [60] has studied the GPD $E$ along the same lines as discussed here.

The GPD $E$ is probed by the transverse target spin asymmetry $^{6}$

$$
A_{U T}^{\sin \left(\phi-\phi_{s}\right)} \sim \operatorname{Im}\left[\langle E\rangle^{*}\langle H\rangle\right],
$$

for given $H$ [8]. The described parametrization of $E$ is consistent with the data on $\rho^{0}$ production from HERMES [76] and COMPASS [77] (see Fig. 14). However, only $E$ for valence quarks matters for $A_{U T}$ since the sea and gluon contribution to $E$ cancel to a large extent. Fortunately the analysis of DVCS data [59] provides additional although not very precise information on $E^{\text {sea }}$. To leading-order of pQCD there is no gluon contribution in DVCS and therefore $E^{\text {sea }}$ becomes visible. The data on the $\sin \left(\phi-\phi_{s}\right)$ modulation of the transverse target spin asymmetry for DVCS measured by the HERMES collaboration [78] are shown on the right hand side of Fig. 14 and compared to the results obtained in [59]. Despite the large experimental errors a negative $E^{\text {sea }}$ seems to be favored. Independent information on $E^{g}$ would be of interest. This may be obtained from a measurement of the transverse target polarization in $J / \Psi$ photoproduction [79].

\section{Ji's sum rule}

The knowledge of the GPDs allow for an evaluation of the angular momenta the partons inside the proton carry. At $\xi=t=0$ the angular momenta ${ }^{7}$ are given by the sum of the second moments of the PDFs and the $\xi=t=0$ limits of $E[80](q=u, d, s, \bar{u}, \bar{d}, \bar{s})$

$$
J^{q}=\frac{1}{2}\left[q_{20}^{q}+e_{20}^{q}\right], \quad J^{g}=\frac{1}{2}\left[g_{20}+e_{20}^{g}\right]
$$

The analysis of the nucleon form factors [18] provides

$$
J_{v}^{u}=0.230_{-0.024}^{+0.009}, \quad J_{v}^{d}=-0.004_{-0.017}^{+0.011}
$$

for the valence quarks. For an evaluation of $J$ for all quarks and the gluon the moments from the ABM11 PDFs are used [29], the results (46) on $J$ for valence quarks and the estimate of $e_{20}^{s}$ from the positivity bound (43) and the analysis of $A_{U T}$ for DVCS [59]: $e_{20}^{s}=0.0 \ldots-0.026$. Assuming a flavor symmetric sea for $E$ one subsequently fixes the $2^{\text {nd }}$ moment of $E_{g}$ from the sum rule (42) $\left(e_{20}^{g}=\right.$ $-0.041 \ldots 0.115)$. Combing all information on the second moments, one obtains at the scale $2 \mathrm{GeV}$

$$
\begin{aligned}
& J^{u+\bar{u}}=0.261 \ldots 0.235, \quad J^{s+\bar{s}}=0.017 \ldots-0.009 \\
& J^{d+\bar{d}}=0.035 \ldots 0.009, \quad J^{g}=0.187 \ldots 0.265 .(47)
\end{aligned}
$$

${ }^{6} \phi_{s}$ is the orientation of the target spin vector with respect to the lepton plane.

${ }^{7}$ For a proton that moves along the 3 -direction, $J$ is the expectation value of the 3-component of the parton angular momentum operator.

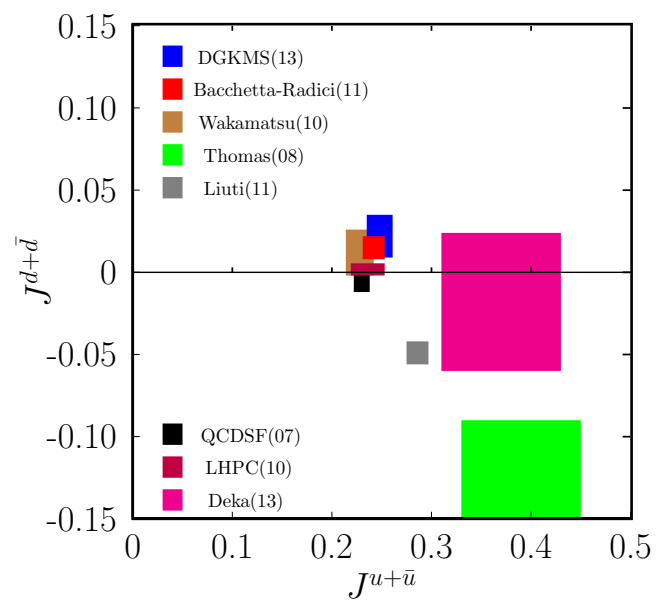

Figure 15. Results on the angular momenta for $u$ and $d$ quarks. Data are from the combined analyses $[18,46,59]$ and from [8187].

The values of the left-hand side are evaluated from $e_{20}^{s}=$ 0.0 , those on the right-hand side from $e_{20}^{s}=-0.026$. Thus, the badly known $E^{s}$ determines the uncertainties of the angular momenta at present. Data on $A_{U T}$ for DVCS with smaller errors than obtained by HERMES [78] would reduce the errors on the angular momenta. The large value of $J^{g}$ is no surprise. The value of $g_{20}$ represents the familiar result that about $40 \%$ of the proton's momentum is carried by the gluons. Since $\left|e_{20}^{g}\right|$ seems to be much smaller than $g_{20}$ this result is not changed much. The angular momenta in (47) sum to $1 / 2$, the spin of the nucleon, because the sum rule (42) is used in the analysis and the PDFs respect the momentum sum rule of DIS. It is to be stressed that the results are obtained from a combination of inclusive (the PDFs) and exclusive data (form factors, DVMP, DVCS). This differs from attempts to understand the nucleon spin only from DIS. A comparison of different results on the angular momenta is made in Fig. 15. There are also experimental results on the angular momenta extracted from DVCS data: $J^{d+\bar{d}}+J^{u+\bar{u}} / 5=0.18 \pm 0.14$ by [88] and $J^{d+\bar{d}} / 2.9+J^{u+\bar{u}}=0.42 \pm 0.21 \pm 0.06$ by HERMES [89]. These results are strongly model-dependent. Among other things they rely on the assumption of proportionality between $e^{q_{v}}$ and $q_{v}$ which is in conflict with the form factor analysis [18] and with perturbative QCD arguments [90]. In a recent lattice QCD study [91] the lowest moment of the isoscalar quark distribution, $u_{20}-d_{20}$, has been calculated for pion masses ranging from 157 till $500 \mathrm{MeV}$. Substantial contributions from excited states to the nucleon structure have been found. After their subtraction the moment $u_{20}-d_{20}$ is much smaller $(\simeq 0.2)$ than obtained in other lattice QCD studies ( $\simeq 0.26$ as for instance in [87]) but still larger than found in PDF analyses $(\simeq 0.16)$, e.g. [29]. Thus, one has to be cautious in applying lattice QCD results, there may still be substantial uncertainties.

The orbital angular momenta are obtained from $J$ by subtracting the first moment of the polarized PDFs

$$
L^{q}=\frac{1}{2}\left[q_{20}+e_{20}^{q}-\Delta q_{10}\right]
$$


Taking the polarized PDFs from [49], one obtains for the valence quarks the values

$$
L_{v}^{u}=-0.141_{-0.033}^{+0.025}, \quad L_{v}^{d}=0.114_{-0.035}^{+0.034},
$$

and for quarks generally

$L^{u+\bar{u}}=-0.146 \ldots-0.172, \quad L^{d+\bar{d}}=0.263 \ldots 0.237$,

$L^{s+\bar{s}}=0.073 \ldots 0.047$.

A reliable decomposition of $\mathrm{J}^{g}$ in spin and orbital angular momentum is not possible at present [92].

\section{Summary}

I have summarized the recent progress in the analysis of hard exclusive leptoproduction of mesons $\left(\rho^{0}, \phi, \omega, \pi^{+}\right)$ and photons at small skewness and small $-t$ within the handbag approach. A set of GPDs has been extracted which is constructed from double distributions with parameters adjusted to meson leptoproduction data $\left(\rho^{0}, \phi\right.$ and $\pi^{+}$) and nucleon form factors. This set of GPDs allows for a parameter-free calculation of DVCS and has also been used in an analysis of $\omega$ leptoproduction. Very good agreement is found with the SDMEs measured by the HERMES collaboration [4]. Interesting predictions have also been given for $\pi^{0}$ and $\eta$ production where the transversity GPDs seem to dominate. Lack of small $\xi$, small $-t$ data prevents a verification of these predictions at present. Large skewness data from JLab6, however, do agree with the predictions in tendency.

There are many observations that the experimental data do not agree with the naive asymptotic results obtained in collinear approximation (leading-twist accuracy) and leading-order of perturbation theory. In particular at JLab6 kinematics, characterized by large skewness and small $W$, the application of the handbag approach is problematic-meson leptoproduction in this kinematical region is not understood as yet. One has to be aware of eventual soft-physics corrections in some of the reactions. In any case an application of the GPDs derived in [8-10] to the region of JLab6 kinematics requires their extrapolation to large $\xi$ and large $-t$.

Acknowledgements: The authors thanks Umberto D'Alesio and Francesco Murgia for the kind invitation to the interesting and excellent organized workshop on Transversity in Chia (Sardinia).

\section{References}

[1] J. C. Collins and A. Freund, Phys. Rev. D59, 074009 (1999).

[2] J. C. Collins, L. Frankfurt and M. Strikman, Phys. Rev. D56, 2982 (1997).

[3] F.D. Aaron et al. [H1 Collaboration], JHEP 1005, 032 (2010).

[4] A. Airapetian et al. [HERMES Collaboration], arXiv:1407.2119 [hep-ex].

[5] S. V. Goloskokov and P. Kroll, Eur. Phys. J. A47, 112 (2011).
[6] A. Airapetian et al. [HERMES Collaboration], Phys. Lett. B659, 486 (2008).

[7] H. P. Blok et al. [Jefferson Lab Collaboration], Phys. Rev. C78, 045202 (2008).

[8] S. Goloskokhov and P. Kroll, Eur. Phys. J. C50, 829 (2007).

[9] S. Goloskokhov and P. Kroll, Eur. Phys. J. C53, 367 (2008).

[10] S. Goloskokhov and P. Kroll, Eur. Phys. J. C65, 137 (2010).

[11] D. Mueller et al, Fortschr. Phys. 42, 101 (1994).

[12] A.V. Radyushkin, Phys. Lett. B449, 81 (1999).

[13] H.N. Li and G.F. Sterman, Nucl. Phys. B381, 129 (1992).

[14] M. Diehl and D. Y. .Ivanov, Eur. Phys. J. C52, 919 (2007).

[15] M.V. Polyakov and C. Weiss, Phys. Rev. D60, 114017 (1999).

[16] I.V. Musatov and A.V. Radyushkin, Phys. Rev. D61, 074027 (2000).

[17] M. Diehl et al., Eur. Phys. J. C39, 1 (2005).

[18] M. Diehl and P. Kroll, Eur. Phys. J. C73, 2397 (2013).

[19] M. Burkardt, Phys. Rev. D 62, 071503 (2000) [Erratum-ibid. D66, 119903 (2002)].

[20] M. Burkardt, Int. J. Mod. Phys. A18, 173 (2003).

[21] M. Vanderhaeghen, P. A. M. Guichon and M. Guidal, Phys. Rev. D60, 094017 (1999).

[22] A. Freund, M. McDermott and M. Strikman, Phys. Rev. D67, 036001 (2003).

[23] M. Meskauskas and D. Müller, Eur. Phys. J. C74, 2719 (2014).

[24] K. Kumerički and D. Mueller, Nucl. Phys. B841, 1 (2010).

[25] A. D. Martin, M. G. Ryskin and T. Teubner, Phys. Rev. D62, 014022 (2000).

[26] L. Frankfurt, W. Koepf and M. Strikman, Phys. Rev. D54, 3194 (1996).

[27] J. Nemchik, N. N. Nikolaev, E. Predazzi and B. G. Zakharov, Z. Phys. C75, 71 (1997).

[28] J. Pumplin et al. [CTEQ collaboration], JHEP 0207, 012 (2002).

[29] S. Alekhin, J. Blumlein and S. Moch, Phys. Rev. D86, 054009 (2012).

[30] S. Chekanov et al. [ZEUS Collaboration], Nucl. Phys. B718, 3 (2005).

[31] S. Chekanov et al. [ZEUS Collaboration], PMC Phys. A1, 6 (2007).

[32] A. V. Vinnikov, hep-ph/0604248.

[33] M. Diehl and W. Kugler, Phys. Lett. B 660, 202 (2008).

[34] A. D. Martin et al, Eur. Phys. J. C35, 325 (2004).

[35] S. Alekhin, JETP Lett. 82, 628 (2005) [Pisma Zh. Eksp. Teor. Fiz. 82, 710 (2005)].

[36] S. A. Morrow et al. [CLAS Collaboration], Eur. Phys. J. A39, 5 (2009).

[37] D. G. Cassel et al, Phys. Rev. D24, 2787 (1981). 
[38] J. P. Santoro et al. [CLAS Collaboration], Phys. Rev. C78, 025210 (2008).

[39] I. Gabdrakhmanov and O. Teryaev, PoS Baldin ISHEPP-XXI, 035 (2012).

[40] B. Pasquini, M. V. Polyakov and M. Vanderhaghen, arXiv:1407.5960 [hep-ph].

[41] A. Airapetian et al. [HERMES Collaboration], Phys. Lett. B682, 345 (2010).

[42] V. M. Braun and I. E. Filyanov, Z. Phys. C48, 239 (1990) [Sov. J. Nucl. Phys. 52, 126 (1990)] [Yad. Fiz. 52, 199 (1990)].

[43] M. Beneke and T. Feldmann, Nucl. Phys. B592, 3 (2001).

[44] L. Mankiewicz, G. Piller and A. Radyushkin, Eur. Phys. J. C10, 307 (1999).

[45] M. Penttinen, M. V. Polyakov and K. Goeke, Phys. Rev. D62, 014024 (2000).

[46] S. Goloskokhov and P. Kroll, Eur. Phys. J. C74, 2725 (2014).

[47] M. Gockeler et al. [QCDSF and UKQCD Collaborations], Phys. Rev. Lett. 98, 222001 (2007).

[48] M. I. Eides, L. L. Frankfurt and M. I. Strikman, Phys. Rev. D59, 114025 (1999).

[49] D. de Florian, R. Sassot, M. Stratmann and W. Vogelsang, Phys. Rev. D80, 034030 (2009).

[50] M. Anselmino et al., Nucl. Phys. Proc. Suppl. 191, 98 (2009).

[51] I. Bedlinsky et al. [CLAS Collaboration], Phys. Rev. Lett. 109, 112001 (2012).

[52] V. Kubarovsky et al, Proceedings of the 4th Workshop " Exclusive reactions at High Momentum Transfer", Newport News, VA USA, 18-21 May 2010

[53] B. Z. Kopeliovich, I. Schmidt and M. Siddikov, Phys. Rev. D89, 053001 (2014).

[54] H. Moutarde, B. Pire, F. Sabatie, L. Szymanowski and J. Wagner, Phys. Rev. D87, no. 5, 054029 (2013).

[55] A. T. Goritschnig, B. Pire and J. Wagner, Phys. Rev. D89, 094031 (2014).

[56] S. V. Goloskokov and P. Kroll, arXiv:1407.1141 [hep-ph].

[57] V. L. Chernyak and A. R. Zhitnitsky, Phys. Rept. 112, 173 (1984).

[58] A. Airapetian et al. [HERMES Collaboration], Eur. Phys. J. C62, 659 (2009).

[59] P. Kroll, H. Moutarde and F. Sabatie, Eur. Phys. J. C73, 2278 (2013).

[60] D. Boer et al., arXiv:1108.1713 [nucl-th].

[61] P. Kroll, M. Schurmann and P. A. M. Guichon, Nucl. Phys. A598, 435 (1996).

[62] M. Diehl and S. Sapeta, Eur. Phys. J. C41, 515 (2005).
[63] V. M. Braun, A. N. Manashov, D. Mueller and B. M. Pirnay, Phys. Rev. D89, 074022 (2014).

[64] F. D. Aaron et al. [H1 Collaboration], Phys. Lett. B681, 391 (2009).

[65] S. Chekanov et al. [ZEUS Collaboration], JHEP 0905, 108 (2009).

[66] A. Airapetian et al. [HERMES Collaboration], JHEP 1207, 032 (2012).

[67] A. Airapetian et al. [HERMES Collaboration], JHEP 1210, 042 (2012).

[68] J. Lachniet et al. [CLAS Collaboration], Phys. Rev. Lett. 102, 192001 (2009).

[69] S. Riordan et al., Phys. Rev. Lett. 105, 262302 (2010).

[70] O. Gayou et al. [Jefferson Lab Hall A Collaboration], Phys. Rev. Lett. 88, 092301 (2002).

[71] A. J. R. Puckett et al., Phys. Rev. Lett. 104, 242301 (2010).

[72] O. V. Teryaev, hep-ph/9904376.

[73] M. Burkardt, Phys. Lett. B582, 151 (2004).

[74] M. Diehl and W. Kugler, Eur. Phys. J. C52, 933 (2007).

[75] S. Goloskokhov and P. Kroll, Eur. Phys. J. C59, 809 (2009).

[76] A. Airapetian et al. [HERMES Coll.], Phys. Lett. B679, 100 (2009).

[77] C. Adolph et al. [COMPASS Coll.], Nucl. Phys. B865, 1 (2012).

[78] A. Airapetian et al. [HERMES Collaboration], JHEP 0806, 066 (2008).

[79] J. Koempel et al., Phys. Rev. D85, 051502 (2012).

[80] X. -D. Ji, Phys. Rev. D 55, 7114 (1997).

[81] A. Bacchetta and M. Radici, Phys. Rev. Lett. 107, 212001 (2011).

[82] M. Wakamatsu, Eur. Phys. J. A44, 297 (2010).

[83] A. W. Thomas, Phys. Rev. Lett. 101, 102003 (2008).

[84] S. K. Taneja, K. Kathuria, S. Liuti and G. R. Goldstein, Phys. Rev. D86, 036008 (2012).

[85] D. Brommel et al. [QCDSF-UKQCD Collaboration], PoS LAT 2007, 158 (2007).

[86] J. D. Bratt et al. [LHPC Collaboration], Phys. Rev. D82, 094502 (2010).

[87] M. Deka et al., arXiv:1312.4816 [hep-lat].

[88] M. Mazouz et al. [Jefferson Lab Hall A Collaboration], Phys. Rev. Lett. 99, 242501 (2007).

[89] Z. Ye [HERMES Collaboration], hep-ex/0606061.

[90] F. Yuan, Phys. Rev. D69, 051501 (2004).

[91] G. S. Bali et al., arXiv:1311.7041 [hep-lat].

[92] D. de Florian, R. Sassot, M. Stratmann and W. Vogelsang, Phys. Rev. Lett. 113, 012001 (2014). 